Instituto Internacional de Investigación y Desarrollo Tecnológico Educativo INDTEC, C.A.

DOI: https://doi.org/10.29394/Scientific.issn.2542-2987.2018.3.8.12.234-247

OAI-PMH: http://www.indteca.com/ojs/index.php/Revista Scientific/oai

\title{
Estudio Sociocrítico de la Educación en las Cárceles Venezolanas
}

Autora: Yudith Coromoto González Castro Universidad Nacional Experimental "Rafael María Baralt", UNERMB

yudithgonzalez@live.com

Valera, Venezuela

\section{Resumen}

El proceso de reflexión científica tiene como propósito generar aportes teóricos derivados de un estudio sociocrítico de la educación de las cárceles venezolanas. Para ello se realizó un análisis de tipo documental, bajo una profunda revisión bibliográfica, a través de la cual se recabó un conjunto de antecedentes para configurar la realidad del hecho de estudio. El proceso socio crítico se realizó bajo la orientación del enfoque paradigmático con una modalidad cualitativa hermenéutica, con la finalidad de organizar las labores de investigación. Como basamento teórico se configuraron teorías clásicas y contemporáneas de la educación andragógica, así como teorías sociológicas que sirven de base para la construcción teórico-científica para el mejorar la práctica educativa de las cárceles venezolanas.

Palabras clave: educación; prisión; sanción penal. 


\section{Sociocritical Study of Education in Venezuelan Cages}

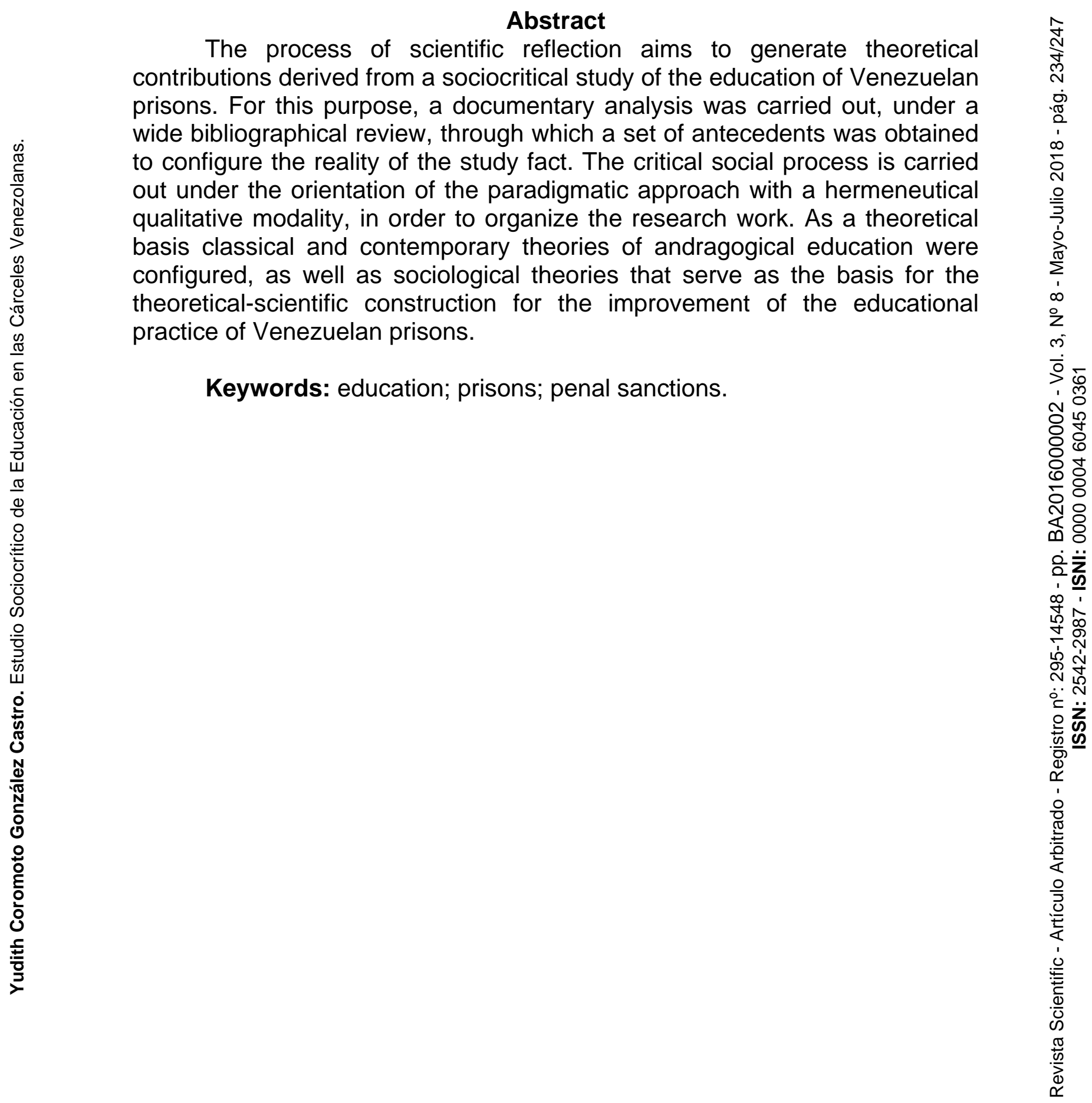

Date Received: 15-06-2017

Date Acceptance: 12-10-2017 


\section{Introducción}

El estudio sociocrítico, se define como un acumulado de doctrinas, bosquejos e hipótesis que reaccionan a lo opuesto de la corriente del positivismo. Tales ideas establecen el requerimiento de promocionar una independencia racional y liberadora para las personas. En opinión de Fernández (2002:18): "es un enfoque investigativo que promueve la participación masiva y la auto-reflexión". A partir de este estudio se estudia la realidad de las cárceles venezolanas en la praxis educativa, donde se contrasta la teoría y la práctica, fundamentándose en el conocimiento, incluyendo la participación de los actores de análisis en el proceso investigativo, así como dirigir el conocimiento hacia la libertad del ser humano.

De acuerdo con el Tesauro de la UNESCO la cárcel significa prisión, calabozo, celda, centro penitenciario, establecimiento penitenciario, presidio, donde un individuo cumple una sanción penal, convirtiéndose en prisionero. Al respecto, las cárceles venezolanas desde la orientación de la (UNESCO, 1998a), son espacios diseñados para alojar a personas que han vulnerado las leyes de la sociedad, cuyo objeto, es reformar a quienes no respetan las normas. En este establecimiento se recluye a los individuos con el fin de castigar tal situación.

Desde este escenario, se torna conveniente analizar la educación en las cárceles venezolanas, según el Tesauro de la UNESCO la educción está definida como un método de aprendizaje, enseñanza y formación a través de diversos medios: aprendizaje en línea, enseñanza presencial, enseñanza por correspondencia, enseñanza a distancia, formación a distancia, tele formación e incluye diversas ciencias de la educación y ambiente educacional.

La educación en las cárceles venezolanas, se manifiesta como un régimen complejo para generar aprendizajes significativos y cambios desde el punto de vista académico, social, cultural y personal en los privados de libertad. En este sentido, la educación en estos recintos no debe estar 
desligada de los principios sociales y políticos comunes que se consideran derechos inalienables. Por lo tanto, la educación debe ser un sistema con igualdad de oportunidades para todas las personas (UNESCO, 1998b), incluyendo a los privados de libertad, que aunque le otorgan igualdad reconocida, ésta es meramente formal y contenida en los textos legales vigentes, pero no es real a lo que se da en el entorno carcelario, dado que sólo se garantiza el acceso a los demandantes educativos, pero no su permanencia, prosecución, incluso graduación y formación de valores.

Esto quiere decir, que el privado de libertad que exija el derecho al estudio se le reconocerá su matriculación, pero dependerá de su esfuerzo y sacrificio poder lograr los objetivos, metas de aprendizaje y restitución de valores. Desde esta perspectiva, no se trata de garantizar las condiciones de permanencia, sino de resistencia para poder sobrevivir dentro del proceso escolar, en donde los progresos que se vayan sucediendo continuamente en la adquisición de conocimientos, experiencias y formas superiores de conducta obedezcan a las condiciones sociales, es decir, que posean madurez perceptiva, motora e intelectual para vencer dificultades y exigencias de los diversos tipos de aprendizajes requeridos para reinsertase de nuevo a la sociedad.

De este modo, el éxito educativo del privado de libertad no está condicionado a solo a los factores de planificación o programación educativa, sino de maduración personal y al factor social para mantener viva la fuerza impulsadora de la motivación de permanecer, proseguir, egresar y restituir valores. Ya que el sistema escolar educativo de las cárceles vigente no está básicamente estructurado para cumplir con las demandas anteriores.

Esto se traduce prácticamente en la necesidad de insertar un paradigma educativo con bases andragógicas, ya que actualmente la educación que se imparte en los recintos, agrede letalmente la eficiencia, la calidad académica que debe preservarse y estimularse en los estudiantes para poder ofrecer 
conocimiento científico, tecnológico y humanístico, al desarrollo integral y autónomo.

Por lo tanto, para cubrir la situación de crisis que viven las cárceles desde el punto de vista educativo, se debe sumar la auténtica intensidad de cambiar la estructura educativa tradicional por una educación andragógica cuya presencia contribuirá a profundizar la deteriorada vida institucional, académica y administrativa del sistema educativo implementada hasta ahora.

En fin, este artículo propone realizar un estudio socio crítico de la educación en las cárceles venezolanas, con el fin de aportar reflexiones que conlleven a mejorar la práctica educativa en estos recintos.

\section{La educación en las cárceles venezolanas}

La educación es relevante en todas las etapas del desarrollo humano, pero alcanza una valiosa importancia cuando sus beneficiarios se remiten a los privados de libertad, puesto que les permite incrementar sus recursos personales en función de su reinserción a la sociedad. Desde esta perspectiva, la educación es un derecho humano esencial para poder entrenar todos los demás derechos, el cual tiene como objeto el desarrollo integral del sujeto. En consecuencia, el acceso de las personas a la educación genera la creación de lazos de pertenencia a la sociedad, es decir, crea la comunicación y recreación de la cultura.

En referencia a lo anterior, el Estado es el encargado de garantizar y promover el goce efectivo de la educación y de todos los derechos humanos, ya que en teoría la persona encarcelada sólo está privada de su libertad ambulatoria, por lo tanto, debe disfrutar y respetársele los demás derechos constitucionales.

De este modo, la educación en las cárceles a nivel latinoamericano está sumergida en una franca crisis, que cada día cobra mayor intensidad y proporción, haciendo cada vez menor la posibilidad de ser sometida a un 
esperado control que permita atenuar su desmesurada profundización, (Tunnermann, 2010). En efecto, la crisis de la educación se define a partir del hecho de la manifiesta incapacidad del sistema educativo de dar, total o parcialmente, satisfacción o respuesta a los problemas concretos planteados, especialmente desde el punto de vista de la restitución de valores.

En esta línea, se concibe que la naturaleza y carácter de la educación, así como la docencia en las cárceles venezolanas, debe dejar de ser un proceso basado en un criterio pedagógico que ignora la condición adulta del estudiante privado de libertad, pues sin exagerar, la mayoría de los docentes está plena o casi totalmente convencidos de que el estudiante en estos centros no se puede someter a una situación de autoritarismo en la que se reduce a su más mínima expresión la posibilidad de participación consciente, creativa y crítica, de acuerdo con su formación integral, desarrollo intelectual, emocional, social y ergológico, (Picón, 2004). Por lo tanto, el sistema educativo debe adentrarse a la luz de la novedosa teoría de educación andragógica o de adultos.

Lo anterior es relevante, porque la educación tradicional que se imparte en las cárceles, por lo general, niegan las capacidades de autorrealización, autonomía, autorresponsabilidad y autodeterminación inherentes a la condición de adulto del estudiante, (Didriksson, 2010): situación ésta que genera un estado de regresión psico-socio-emocional en el que debería ser sujeto del proceso, ya que al obligarle a someterse a los moldes rígidos propios de una estructura escolarizada al rigor del pedagogísmo más alucinante como la educación tradicional, termina por resignarse a ser aquel que debe practicar una conducta impuesta, adquiriendo un patrón para poder pensar y actuar en la toma de decisiones y asumir responsabilidades en la vida social.

La presencia de esta realidad en la vida educativa de los privados de libertad conduce inexorablemente a que ellos irrespeten su derecho a formarse como personas íntegras e internalicen el enajenante espíritu de sumisión e 
irrespeto, que significa la renuncia a ser un hombre integral dueño de su presente y de su futuro. Por consiguiente, es un error no intentar algo para vencer, aunque sea parcialmente, esta situación regida por los principios de los antivalores. No hacer nada equivale a mantener una de las causas que determinan la crisis académica de las cárceles, la de seguir siendo ineficaz y poco formativa de valores.

En consecuencia, mientras que los que conforman la comunidad educativa de las cárceles no adquieran conciencia de esta realidad inobjetable, se verá imposibilitado asumir la condición de agentes de cambio en la renovación escolar, para ello, los programas de educación son los medios considerables para renovar y perfeccionar el ejercicio docente y la estructura organizativa educativa en base a una educación andragógica, con el fin de mejorar la capacidad de formación, reconstruir lo que hoy prevalece para alcanzar a crear y establecer una educación deslastrada del peso de la tradición sin temor al futuro, sobre la base de los principios andragógicos que permitirán adecuar las relaciones interpersonales (docente - estudiante, estudiante - estudiante, docente - autoridad), los recursos de aprendizaje, los contenidos, los objetivos, las estrategias metodológicas, en fin el currículum a la naturaleza bio-psico-social de la los miembros de la comunidad estudiantil de la cárcel.

Desde este punto de vista, el programa educativo de las cárceles debe estar integrado por un conjunto de asignaturas que aporten el conocimiento de las ideas, teorías, escuelas y disciplinas que conforman la teoría andragógica y sus enfoques filosóficos, antropológicos, psicológicos, sociológicos, políticos. El cual tiene que estar dirigido a una revisión analítica - critica de las diversas teorías educativas relacionadas con la educación en el país, pero también con el entorno personal de los privados de liberad, para que de esta manera se constituya un plano teórico - práctico - crítico de la formación de los individuos y de los docentes. 
Asimismo, deben aportar conocimientos en primer lugar sobre la teoría de la tecnología educativa, abarcando el diseño curricular e instruccional, además de incluir el entrenamiento práctico en las técnicas y metodologías de la tecnología educativa como parte importante en la formación andragógica. En segundo lugar, se tiene que incluir la teoría y el entrenamiento relativo a la metodología de la investigación y sus diferentes modalidades y aplicaciones, todo este conjunto conforma el plano tecnológico (no tecnocrático) y heurístico de la formación docente del profesor.

Como se puede apreciar, la educación andrológica procura tomar en cuenta todos los componentes que abarca la formación de los privados de libertad para que se convierta en una actividad donde el proceso de enseñanza aprendizaje pueda ser calificado con una continuidad y progresividad horizontal para lograr una unidad didáctica que integre e involucre a todos los participantes.

De esta manera, los programas educativos y la actualización andragógica de los docentes y estudiantes recomponen la condición que ha sido causa de la ausencia de una práctica legítima de la autonomía como condición necesaria para el ejercicio pleno de la vida educativa. De hecho se puede decir, sin pecar de simplicidad e ingenuidad, que los docentes, junto con los directivos y personal del penal al asumir una actitud autoritaria frente al estudiante se ha permitido que no se haga presente la condición de valores en los adultos privados de libertad, que no es otra cosa que impedirle que asuma responsablemente la posibilidad de participar racional, creativa y críticamente en la formación educativa y de valores, lo que ha llevado que la autonomía se haya convertido en un auténtico fetiche sin realización plena y concreta en la educación carcelaria.

Esto se debe a que la naturaleza y ejecución de la educación autónoma requiere indiscutiblemente de una base social adulta, es decir, de una comunidad de adultos que, conformada en torno al logro de finalidades 
superiores deseables, no sólo de carácter formativos-profesional sino también de investigación y de solución de problemas vitales, estimule la conciencia crítica y la capacidad creadora en todos para que sean aptos en el futuro desempeño de los adultos educativos.

Dicho de otra manera, el no contar con una educación con una base andragógica, generará que los estudiantes no estén en condición de realizar mejor su desarrollo dinámico y creador al no tener la posibilidad de fomentar y mantener su desarrollo académico y sus valores éticos morales.

De allí, se desprende la importancia que asume la actual organización estructural académica del sistema educativo carcelario y su funcionamiento, puesto que se requiere de la adopción de la praxis subyacente en el modelo andragógico (praxiología andragógica). Por tanto, se hace impostergable que el personal docente tenga un ejercicio centrado en desarrollar una interpretación racional de la naturaleza del aprendizaje, inmersa en la formación de valores y de la función que debe cumplir para insertar apropiadamente a los estudiantes privados de libertad a la sociedad. Por ello, deben asumirse ciertas medidas con el objeto de renovar y modernizar las viejas estructuras que sirven de piso y marco de la educación en las cárceles.

Es así como la educación en las cárceles debe dirigir su acción a la capacitación, actualización andragógica del personal docente, (Marcelo; 1995); asimismo debe dotarse de una capacidad superior para enfocar y resolver los problemas educativos que el aprendizaje de adultos plantea, también debe procurar establecer una formación que estimule y profundice el desarrollo de la ciencia andragógica.

\section{Abordaje Metodológico}

El contexto de la investigación se circunscribe a la realidad de la educación en las cárceles venezolanas desde una perspectiva axiológica de la reinserción de los privados de libertad a la sociedad, el abordaje 
metodológico se realizó desde un punto de vista documental, critico reflexivo.

Delimitado el contexto de análisis, se seleccionaron como actores de estudio a las cárceles venezolanas y sus programas educativos, permitiendo establecer la precisión de la información. La recolección de datos se realizó a través de la técnica de revisión documental y apoyo en las fuentes primarias como declaraciones, artículos, análisis sobre la realidad educativa en las cárceles, así como en libros, información Web, opiniones de expertos, entre otros.

El análisis socio crítico se realizó contrastando la información documental, con las opiniones de expertos y la realidad de la educación en las cárceles, generando conocimiento y concretar reflexiones que ayuden a mejorar la calidad de la educación de los privados de libertad en Venezuela.

\section{Conclusiones}

La educación en las cáceles venezolanas enfrenta un desafío conjunto de elementos coyunturales, económicos y sociales como: elevar la calidad en los procesos educativos, recursos económicos en menor cuantía de lo exigido; mejorar la calidad académica con relación a lo presupuestario, financiero y administrativo. Por lo tanto, esta investigación se armonizó con la agenda cambiante de la gestión de las cárceles venezolanas, donde se plantea la incorporación de herramientas novedosas de autoevaluación y autorregulación de las funciones educativas. Es conveniente decir, que la idea de una educación de adultos en sus diversas modalidades y niveles, formales, o no formales, debe ser siempre considerada para que los estudiantes desarrollen y enriquezcan sus aptitudes, sus conocimientos, sus competencias y sus actitudes, lo cual tendría repercusiones inestimables en el avance y desarrollo educativo, al superar los límites que impone el pedagogísmo paroxístico existente, como de la vida social circundante, al participar en los procesos de valoración humana para promover los cambios estructurales de 
la sociedad, (García, 2007).

Desde este contexto, también se debe tener presente que otro de los aspectos presentes en la crisis de la educación carcelaria es el personal docente, el cual muy poco se ha capacitado desde el criterio de los principios andragógicos para impartir una educación más perceptiva a los privados de libertad. Se debe hacer hincapié que la formación del docente debe estar adaptada a las necesidades del sistema educativo carcelario, puesto que son una necesidad, ya que constituyen un factor determinante de la calidad educativa que se espera.

Por lo tanto, es obvio que la efectividad y eficiencia de la labor educativa andragógica formal para cumplir con la función de contribuir con la enseñanza aprendizaje y restitución de valores de los privados de libertad, depende fundamentalmente del personal docente, así como de la adecuada planificación y supervisión del proceso educativo, (Corredor, 2009).

En otro orden, no se debe perder de vista que los privados de libertad son, ante todo, sujetos de derechos. Y el Estado debe ser garante del cumplimiento efectivo sus derechos. Por ello, los directivos y personal que hacen vida activa como docentes se deben involucrar en la realidad que viven las cárceles para evitar la vulneración de los derechos humanos y ayudar a los privados a prepararse para enfrentarse como personas íntegras a la sociedad.

Se reconoce que la mayoría de los recluidos en las cárceles pertenecen a los sectores más vulnerables de la sociedad. Por tanto, la educación se transforma en un derecho que reconoce los demás derechos, sin embargo, esta realidad, no es verdadera, ya que la educación en ocasiones ha sido negada o mal impartida en la cárcel.

En consecuencia, el abordaje institucional sobre la educación en el contexto de la cárcel, debe ser una invitación de reflexión sobre el para qué, el por qué y cómo la educación debe fortalecerse como derecho y no como un beneficio, a fin de ser una reflexión que contribuya al diseño de una apropiada 
política pública que haga del proceso educativo un espacio social que forme profesional y personalmente a los privados de libertad.

En fin, los hallazgos de este estudio contribuyen a la interpretación y comprensión de la educación en las cárceles, como un aporte para generar un sistema educativo eficaz y sólido que permita reinsertar personas íntegras a la sociedad.

\section{Referencias}

Corredor, J. (2009). Las metas de la educación. Una propuesta de transformación consejo de desarrollo científico y humanístico. Caracas; Venezuela: Universidad Central de Venezuela.

Didriksson, A. (2010). La educación y la innovación. Caracas, Venezuela: Ediciones IESALC/UNESCO.

Fernández, A. (2002). Filosofía, investigación, innovación y buenas prácticas. México: Editorial UNAM.

García, G. (2007). Conocimiento, educación y sociedad en América Latina. Caracas, Venezuela: Cendes/Editorial Nueva Sociedad.

Marcelo, C. (1995). La formación del profesorado para el cambio educativo. Barcelona, España: EUB. [Libro en línea]. Recuperado de: https://www.researchgate.net/profile/Carlos Marcelo/publication/25619 4929 Formacion del Profesorado para el Cambio Educativo/links/0 2e7e521f1a3f866d2000000/Formacion-del-Profesorado-para-elCambio-Educativo.pdf

Picón, G. (2004). El proceso de convertirse en educador. Ediciones de la Caracas, Venezuela: Universidad Pedagógica Experimental Libertad.

Tunnermann, B. (2010). La educación en América latina. Bogotá, Colombia: En Universitas.

Tesauro de la UNESCO (1977). Información de vocablos. UNESCO. Recuperado de: http://vocabularies.unesco.org/browser/thesaurus/es/ 
UNESCO (1998a,b). Declaración mundial sobre la educación en el siglo XXI. Visión y Acción y Marco de Acción Prioritaria para el Cambio y el Desarrollo de la Educación superior. París, Francia: Conferencia Mundial sobre la Educación Superior. Recuperado de:

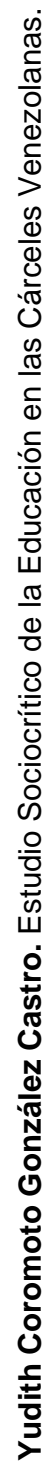
http://www.unesco.org/education/educprog/wche/declaration spa.htm 


\section{Yudith Coromoto González Castro \\ e-mail: yudithgonzalez@live.com}

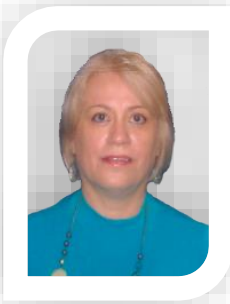

Nacida en Valera estado Trujillo, Venezuela. Soy Doctora en Educación de la Universidad Nacional Experimental "Rafael María Baralt" (UNERMB), MSc. en Docencia para la Educación Superior (año 2008), de la Universidad Nacional Experimental "Rafael María Baralt" (UNERMB), MSc. en Educación Mención Gerencia Educacional (año 2003), de la Universidad Pedagógica Experimental Libertador (UPEL), Diplomado en Semiótica del Núcleo Universitario "Rafael Rangel" (NURRULA), Diplomado en Metodología del Convenio Núcleo Universitario "Rafael Rangel" (NURR-ULA) y la Universidad Pedagógica Experimental Libertador (UPEL).

Ingrese al M.P.P.S.P. el día 06 de marzo del 2012, Mediante Transferencia de la Dirección Nacional del Servicio Penitenciario al Ministerio del Poder Popular para el Servicio Penitenciario, según Decreto N. 8.828 de fecha 06 de marzo del 2012, publicado en la Gaceta Oficial de la República Bolivariana de Venezuela N. 39877 de fecha 06 de marzo del 2012.

Actualmente laboro en el Centro Penitenciario de la Región Andina (CPRA), Mérida, Venezuela, con 11 años de servicio.

El contenido de este manuscrito se difunde bajo una Licencia de Creative Commons ReconocimientoNoComercial-Compartirlgual 4.0 Internacional 